\title{
The role of physiotherapy in the management of vulvodynia
}

Vulvodynia is an umbrella term used to describe pain or discomfort in the vulva, lasting for more than three months and for which no obvious aetiology can be found (Bornstein et al 2016). Understandably, the condition creates significant physical, psychological, and emotional issues for the woman affected and her intimate partner.

The clinical presentation of women with vulvodynia varies greatly, which means that taking a thorough history and performing a careful examination is essential for the interpretation of the presentation and for ruling out differential diagnoses of vulval pain (Cox and Neville 2012). This variability also means that there is no set recipe for managing someone with vulvodynia: each woman needs an individualised assessment and intervention plan that addresses the specific biological, psychological, and social factors contributing to her pain, and the impact of her pain on her life (Chalmers 2015).

Although physiotherapists are often the first point of contact for a woman with vulvodynia, it is important to also get a medical assessment because there are several conditions that can present as, or alongside, vulvodynia. Differential or co-existing diagnoses include dermatological conditions, both benign and malignant, vulvovaginal infections, and pudendal neuralgia. Where coexisting diagnoses have been excluded or effectively managed, physiotherapy can be effective in reducing pain and improving quality of life in women with vulvodynia (for example, Bergeron et al (2002), Goldfinger et al (2009), Hartmann et al (2007)). Due to the complex nature of vulvodynia, physiotherapists treating women with this condition should have advanced education in the field of women's health. Here we outline the evidence-based recommendations for the physiotherapy management of women presenting with vulvodynia.

\section{Recommendation 1: Validation}

Often the first step on a woman's road to recovery is having her pain validated as being real (Sadownik 2014). This validation can bring comfort and counter any thoughts she may have that the condition is imagined or 'all in her head'. It is often helpful to have the woman's partner present during these conversations to further legitimise her pain experience. Labeling the pain as vulvodynia validates that the pain exists but is not driven by sinister or harmful pathology. Evaluating the impact of pain on the individual's life (Chalmers 2015) is also validating and, critically, important for planning management.

\section{Recommendation 2: Education}

Patient education is considered best-practice for many pain conditions, including back pain (Reese and Mittag 2013), neck pain (Yu et al 2014), and fibromyalgia (Ablin et al 2013). While several narrative reviews advocate patient education for the treatment of vulvodynia (Cox and Neville 2012, Haefner et al 2005, Mandal et al 2010), the exact content of this education is vague. Previous research has highlighted the efficacy of pain biology education - so called 'explaining pain' - in patients with chronic low back pain (Moseley et al 2004). While no such research has been conducted in women with vulvodynia, there are similarities that exist between low back pain and vulvodynia, for example both are not usually associated with structural pathology; both involve changes in the central nervous system; and both benefit from a biopsychosocial approach. Best practice management of vulvodynia currently includes pain biology education that aims to increase the woman's understanding of the biological mechanisms underpinning their condition and to reduce the perceived threat associated with their pain.

Education on simple management techniques can also be beneficial (Goldstein and Burrows 2008). Women can be advised to avoid irritants (soaps or body washes, vaginal douching, having bubble baths, and using scented sanitary pads or tampons (Edwards 2003, Glazer and Ledger 2002)). The vagina has its own cleaning and $\mathrm{pH}$ regulatory mechanisms, mediated by a large amount of 'good' bacteria. There is no need to clean the inside of the vagina and the external vulva simply requires washing with warm water and patting dry with a towel. Moisturising the vulva after washing with a non-perfumed cream is recommended in clinical guidelines (Haefner et al 2005, Henzell and Berzins 2015), although supportive clinical studies are lacking.

Addressing a woman's sexual concerns is essential. There is no consensus on whether the best approach is to remain sexually active or to abstain, and it may differ from woman to woman. Abstinence may help patients to avoid aggravating factors but can also take a toll on intimate relationships. If a patient remains in a penetrative sexual relationship, they can be advised use olive or coconut oil as a lubricant because they are free of synthetic materials or perfumes.

\section{Recommendation 3: Stress and anxiety reduction}

Women with vulvodynia often relate the onset or exacerbation of their symptoms to stress and anxiety (Arnold et al 2006, Ehrström et al 2009). Relaxation techniques, in particular progressive relaxation, has positive physiological effects on those suffering from anxiety (Borkovec and Sides 1979). Most clinical guidelines on vulvodynia recommend relaxation (Haefner et al 2005, Mandal et al 2010, Reed 2006), although the exact method or dosage remains unclear. We recommend a gentle progressive muscle relaxation technique that includes the pelvic floor muscles (for example, the audio guide available from http://www.patricianeumann.com.au).

\section{Recommendation 4: Pelvic floor muscle down-training}

Women with vulvodynia often have pelvic floor muscle dysfunction (PFMD). Pelvic floor resting muscle tone is higher and contractions are slower and weaker in women with vulvodynia than in healthy women (Glazer et al 1998, White et al 1997). PFMD can be assessed by using a 1-finger digital examination of the muscles and/or by using biofeedback. If PFMD is observed, the aim of the intervention should be to reduce resting muscle tone. This is achieved through 'downtraining' of the pelvic floor muscles, and can be performed independently or with the use of biofeedback. 
Down-training the pelvic floor is difficult for most women. There are different techniques that can be used and not all women will respond to the same technique. Gentle contract-relax exercises, such as those used in a progressive muscle relaxation technique, can be helpful in improving a woman's proprioceptive awareness of her pelvic floor muscles (Bergeron et al 2002). Maximal pelvic floor muscle contractions, such as those made popular by Kegel (1948), are not supported. The focus of exercises should be on the relaxation of the pelvic floor muscles; a gentle, sub-maximal contraction followed by relaxation can be helpful for women to perceive the sensation of the pelvic floor relaxing. However, if these contractions evoke pain they should be avoided, with exercises focusing solely on relaxation. Specific dosage for pelvic floor down-training exercises is vague; however, two 20-minute sessions per day are often recommended (Edwards 2003, Hollis 2000).

Intravaginal biofeedback is a helpful addition to pelvic floor down-training exercises because it allows patients to visualise their muscle activity (Bergeron et al 2002). It is especially useful in its portability and affordability; patients can complete their pelvic floor down-training exercises at home and receive immediate feedback on their performance (Edwards 2003).

\section{Recommendation 5: Referral}

An effective management plan for vulvodynia will include addressing all of the biological, psychological, and social factors that contribute to a woman's pain. To address these factors appropriately, physiotherapists should not work alone: clinical guidelines recommend a multidisciplinary approach involving physiotherapists, medical doctors, gynaecologists, pain specialists, and counselors or psychologists (Mandal et al 2010).

\section{Other clinical treatments}

There are three other commonly used techniques for treating women with vulvodynia for which there is little supporting evidence: pelvic floor muscle release, vaginal dilators, and electrical stimulation. While their efficacy is unknown, it would seem prudent to consider the potential risks or side effects and the pressing need for further research. Pelvic floor muscle release has been used in combination with other treatments such as biofeedback and relaxation with good patient outcomes (Bergeron et al 2002); however, it is difficult to surmise the actual effect of the soft-tissue release technique. Relevant here is that the technique requires the insertion of a digit which is often painful for women with vulvodynia (Foster et al 2009) and the evidence to suggest that deep soft-tissue massage has no effect on the targeted effect - flexibility of soft-tissue (for example, Thomson et al (2015)). Despite these limitations, deep soft-tissue release remains a popular technique used to treat vulvodynia. Theoretically, vaginal dilators may be helpful to desensitise the vaginal introitus and allow women to regain confidence in engaging in sexual intercourse (Wylie et al 2004). However, again, there is currently little empirical support for this theory. There is one randomised controlled trial investigating the efficacy of transcutaneous electrical nerve stimulation (TENS) in the treatment of vestibulodynia, a subtype of vulvodynia (Murina et al 2008). That trial showed significantly improved pain scores in the TENS group versus the sham treatment group; however, these findings are yet to be replicated. The broad picture that emerges when available treatments for vulvodynia are appraised is one of desperate need for rigorous clinical studies and frank appraisal of pathophysiological models, some of which are retrofitted to apparently effective interventions, some have been disproved and some, one might suggest, are nonsense.

\section{Summary}

The complex nature of vulvodynia can cause frustration for patients and clinicians alike. Physiotherapy can be an effective treatment for vulvodynia, although the specific techniques used to treat the condition can vary widely and much remains to be elucidated with regards to why some treatments seem to work. As the evidence currently stands, management plans for women with vulvodynia should be individualised and target the specific factors contributing to the individual's pain presentation, itself dependent on a thorough biopsychosocial assessment. Key components of a physiotherapy intervention will usually involve: (1) validating the pain as real, (2) educating women on pain biology and simple management techniques, (3) relaxation for stress and anxiety management, (4) pelvic floor muscle down-training, and (5) integration of care with other health professions, such that physiotherapists work alongside others professions to ensure that the specific biological, psychological, and social factors contributing to each woman's pain experience are addressed.

K Jane Chalmers B. Phty (Hons)

Lecturer in Physiotherapy, School of Science and Health, Western Sydney University, Sydney, Australia; Sansom Institute for Health Research, University of South Australia, Adelaide, Australia

Mark R Hutchinson PhD (Med), BSc (Hons)

Professor in the School of Medicine, The University of Adelaide, Adelaide, Australia; Director of ARC Centre of Excellence for Nanoscale BioPhotonics, Australia

G Lorimer Moseley PhD, FACP

Professor of Clinical Neurosciences and Foundation Chair in Physiotherapy, University of South Australia; Sansom Institute for Health Research, University of South Australia, Adelaide, Australia

doi: 10.15619/nzjp/44.3.01

\section{ADDRESS FOR CORRESPONDENCE}

K Jane Chalmers, Physiotherapy, School of Health and Science, Western Sydney University, Locked Bag 1797, Penrith, NSW 2751, Australia.

Email: j.chalmers@westernsydney.edu.au

\section{REFERENCES}

Ablin J, Fitzcharles M-A, Buskila D, Shir Y, Sommer C, Häuser W (2013) Treatment of fibromyalgia syndrome: Recommendations of recent evidence-based interdisciplinary guidelines with special emphasis on complementary and alternative therapies. Evidence-Based Complementary and Alternative Medicine Article ID 485272.

Arnold LD, Bachmann GA, Kelly S, Rosen R, Rhoads GG (2006) Vulvodynia: Characteristics and associations with co-morbidities and quality of life. Obstetrics and Gynecology 107(3): 617. 
Bergeron S, Brown C, Lord M-J, Oala M, Binik YM, Khalifé S (2002) Physical therapy for vulvar vestibulitis syndrome: A retrospective study. Journal of Sex and Marital Therapy 28(3): 183-192. doi:10.1080/009262302760328226.

Borkovec T, Sides JK (1979) Critical procedural variables related to the physiological effects of progressive relaxation: A review. Behaviour Research and Therapy 17(2): 119-125.

Bornstein J, Goldstein AT, Stockdale CK, Bergeron S, Pukall C, Zolnoun D, Coady D (2016) 2015 issvd, isswsh and ipps consensus terminology and classification of persistent vulvar pain and vulvodynia. Obstetrics and Gynecology 127(4): 745-751.

Chalmers K, Catley, MJ, Evans, SF, Moseley, GL (2015) Developing a reliable measure of the impact of pelvic pain: The pelvic pain impact questionnaire (ppiq)

Cox KJ, Neville CE (2012) Assessment and management options for women with vulvodynia. Journal of Midwifery and Women's Health 57(3): 231240

Edwards L (2003) New concepts in vulvodynia. American Journal of Obstetrics and Gynecology 189(3, Supplement): S24-S30. doi:http://dx.doi. org/10.1067/S0002-9378(03)00790-7.

Ehrstrōm S, Kornfeld D, Rylander E, Bohm-Starke N (2009) Chronic stress in women with localised provoked vulvodynia. Journal of Psychosomatic Obstetrics and Gynecology 30(1): 73-79.

Foster DC, Kotok MB, Huang LS, Watts A, Oakes D, Howard FM, Stodgell CJ, Dworkin RH (2009) The tampon test for vulvodynia treatment outcomes research: Reliability, construct validity, and responsiveness. Obstetrics and Gynecology 113(4): 825-832. doi:10.1097/AOG.0b013e31819bda7c.

Glazer H, Jantos M, Hartmann E, Swencionis C (1998) Electromyographic comparisons of the pelvic floor in women with dysesthetic vulvodynia and asymptomatic women. The Journal of Reproductive Medicine 43(11): 959962.

Glazer HI, Ledger WJ (2002) Clinical management of vulvodynia. Reviews in Gynaecological Practice 2(1): 83-90.

Goldfinger C, Pukall CF, Gentilcore-Saulnier E, McLean L, Chamberlain S (2009) Original research-pain: A prospective study of pelvic floor physical therapy: Pain and psychosexual outcomes in provoked vestibulodynia. The Journal of Sexual Medicine 6(7): 1955-1968.

Goldstein AT, Burrows L (2008) Continuing medical education: Vulvodynia (cme). The Journal of Sexual Medicine 5(1): 5-15. doi:10.1111/j.17436109.2007.00679.x.

Haefner HK, Collins ME, Davis GD, Edwards L, Foster DC, Hartmann EDH, Kaufman RH, Lynch PJ, Margesson LJ, Moyal-Barracco M (2005) The vulvodynia guideline. Journal of Lower Genital Tract Disease 9(1): 40-51.

Hartmann D, Strauhal M, Nelson CA (2007) Treatment of women in the united states with localized, provoked vulvodynia: Practice survey of women's health physical therapists. Journal of Women's Health Physical Therapy 31(3): 34-38.
Henzell H, Berzins K (2015) Localised provoked vestibulodynia (vulvodynia): Assessment and management. Australian Family Physician 44(7): 460

Hollis $\mathrm{H}$ (2000) Conservative management of female patients with pelvic pain. Urologic Nursing 20(6): 393

Kegel $\mathrm{AH}$ (1948) Progressive resistance exercise in the functional restoration of the perineal muscles. American Journal of Obstetrics and Gynecology 56(2): 238-248

Mandal D, Nunns D, Byrne M, McLelland J, Rani R, Cullimore J, Bansal D, Brackenbury F, Kirtschig G, Wier M (2010) Guidelines for the management of vulvodynia. British Journal of Dermatology 162(6): 1180-1185

Moseley GL, Nicholas MK, Hodges PW (2004) A randomized controlled trial of intensive neurophysiology education in chronic low back pain. The Clinical Journal of Pain 20(5): 324-330.

Murina F, Bianco V, Radici G, Felice R, Di Martino M, Nicolini U (2008) Transcutaneous electrical nerve stimulation to treat vestibulodynia: A randomised controlled trial. BJOG: An International Journal of Obstetrics and Gynaecology 115(9): 1165-1170

Reed BD (2006) Vulvodynia: Diagnosis and management. American Family Physician 73(7): 1231-1238.

Reese C, Mittag O (2013) Psychological interventions in the rehabilitation of patients with chronic low back pain: Evidence and recommendations from systematic reviews and guidelines. International Journal of Rehabilitation Research 36(1): 6-12

Sadownik LA (2014) Etiology, diagnosis, and clinical management of vulvodynia. International Journal of Women's Health 6 .(1): 437-449. doi:10.2147/IJWH.S37660

Thomson D, Gupta A, Arundell J, Crosbie J (2015) Deep soft-tissue massage applied to healthy calf muscle has no effect on passive mechanical properties: A randomized, single-blind, cross-over study. BMC Sports Science, Medicine and Rehabilitation 7(1): 1.

White G, Jantos M, Glazer H (1997) Establishing the diagnosis of vulvar vestibulitis. The Journal of Reproductive Medicine 42(3): 157-160.

Wylie K, Hallam-Jones R, Harrington C (2004) Psychological difficulties within a group of patients with vulvodynia. Journal of Psychosomatic Obstetrics and Gynecology 25(3-4): 257-265.

Yu H, Côté P, Southerst D, Wong JJ, Varatharajan S, Shearer HM, Gross DP, van der Velde GM, Carroll LJ, Mior SA (2014) Does structured patient education improve the recovery and clinical outcomes of patients with neck pain? A systematic review from the ontario protocol for traffic injury management (optima) collaboration. The Spine Journal [In Press] 